\title{
ALERGIA AO LÁTEX E À BANANA EM CRIANÇAS COM MIELOMENINGOCELE NA CIDADE DO RIO DE JANEIRO
}

\author{
Marta Machado*, Clemax Sant'anna, Vera Aires, Pedro Paulo Rodrigues, Maria Fernanda Pinheiro, Marisa Teixeira \\ Trabalho realizado na Fundação Técnico-Educacional Souza Marques, Laboratório Sérgio Franco \\ e Universidade Federal do Rio de Janeiro, Rio de Janeiro, RJ.
}

RESUMO - Recentemente, reações de hipersensibilidade do tipo I ao látex foram relatadas com freqüência, principalmente nos indivíduos cujo contato com produtos de látex é íntimo e freqüente. Crianças com mielomeningocele (MMC) são as que mais se sensibilizam ao látex, pelo contato freqüente e precoce com este material. Reações alérgicas cruzadas entre alimentos são conhecidas há anos. Atualmente, a alergia ao látex é freqüentemente associada à alergia a frutas como abacate, banana, kiwi, grapefruit, pêssego, papaia e castanha.

Овјетtvos. 0 objetivo deste trabalho consistiu em identificar o número de pacientes com MMC e sensibilização clínico-laboratorial à banana e ao látex.

Métodos. Questionários foram aplicados a 33 crianças com MMC, e sangue foi colhido de 30 para ser efetuada a dosagem de imunoglobulina E (IgE) específica (RAST Pharmacia) para látex, e de 29 para banana. Foi considerada positiva a IgE específica igual ou superior à classe $\mathrm{I}$.

Resultados. Quatro crianças relataram histórias de urticária com látex, e uma criança descreveu urticária e diarréia com banana. 14/30 (46,6\%) apresentavam IgE específica para látex positiva, e $4 / 29$ (I3,7\%) para banana. Nenhum cruzamento de variáveis foi estatisticamente significativo com a sensibilização clínico labora. torial ao látex e à banana.

ConclusöEs. Identificou-se elevada prevalência de alergia ao látex e à banana no grupo de pacientes com MMC. No Brasil necessitamos de mais estudos para analisar a prevalência de reações alérgicas cruzadas entre alimentos e látex em crianças com MMC.

Unitermos: Látex. Banana. Sensibilização. Criança. Mielomeningocele.

\section{INTRODUÇÃO}

Durante os últimos I 5 anos as reações de hipersensibilidade do tipo I (imediato) ao látex da borracha natural têm sido relatadas com uma freqüência maior, principalmente nos indivíduos cujo contato com os produtos de látex (luvas cirúrgicas, catéteres, válvulas, preservativos e chupetas) é íntimo e freqüente!.

Hoje está bem claro que as crianças que apresentam MMC são as que mais se sensibilizam ao látex pelo contato muito freqüente e precoce com catéteres, luvas, derivações ventrículo-peritoneais, entre outros. Esta população é considerada atualmente a de maior risco em apresentar a alergia ao látex, com vários estudos nos países desenvolvidos determinando a sua freqüência, que varia de II,5\% a $72 \%$.

Dois trabalhos publicados na América Latina têm demonstrado uma prevalência de alergia ao látex muito inferior aos estudos

\footnotetext{
* Correspondência:

Rua Visconde de Pirajá, 39 - Ap. 102

Cep: 22410-001 - Ipanema - RJ

martam@openlink.com.br
}

publicados na Europa e Estados Unidos: 4,3\% na Venezuela ${ }^{3}$ e 2,97\% na Argentina ${ }^{4}$; a causa para este fato ainda é desconhecida. Outro estudo argentino encontrou uma incidência de $29 \%$ de hipersensibilidade ao látex em crianças com MMC ${ }^{5}$. Dois estudos recentes, realizados no México, encontraram freqüências de sensibilização ao látex de 4,76\% em crianças atópicas ${ }^{6}$, e de $22 \%$ de pacientes adultos com antecedentes profissionais médicos ou paramédicos ${ }^{7}$.

Atualmente, a alergia ao látex vem sendo freqüentemente associada à alergia a frutas e legumes como abacate, banana, kiwi, grapefruit, papaia, castanha, nozes, ananás, melão, figo, maracujá, tomate, tâmara, batata e pêssego, com freqüências de sensibilização clínicolaboratorial variando de $18,4 \%$ a $32,1 \%$ dos pacientes alérgicos a láte ${ }^{8,9}$.

Os estudos utilizando métodos de inibição com o RAST comprovaram a existência de uma sensibilização cruzada entre o látex e a banana $^{10}$. Estudos de prevalência de ambas as alergias têm sido publicados. Kim" demonstrou que $18,3 \%$ das crianças com MMC apresentavam alergia a banana, e Beezhold ${ }^{12}$ encontrou 38\% de pacientes alérgicos a látex com testes cutâneos de puntura positivos para banana. Em outro estudo, Delbourg ${ }^{13}$ encontrou $50 \%$ de pacientes alérgicos a látex com uma história clínica de alergia a banana, 36\% com testes cutâneos de puntura positivos para banana, e 89\% apresentavam RAST positivo para banana.

Este trabalho pretende contribuir com maiores informações sobre este tema, principalmente no que se refere à sensibilização clínico-laboratorial ao látex e à banana, em crianças portadoras de MMC, na cidade do Rio de Janeiro.

\section{Métodos}

Esta pesquisa foi realizada no Instituto Fernandes Figueira (IFF), pertencente à Fundação Oswaldo Cruz (FIOCRUZ), órgão público federal, situado no Rio de Janeiro, RJ, no período de setembro de 1998 a janeiro de 1999. Foram aplicados questionários aos pais e responsáveis, que respondiam aos mesmos após lerem o Termo de Consentimento Informado onde thes era explicado, em linguagem coloquial e clara, em que consistia o estudo. 


\begin{tabular}{cccc}
\hline \multicolumn{3}{c}{ Tabela I - Distribuição por idade de crianças com mielomeningocele. Iff, 1996-1999 } \\
\cline { 2 - 3 } \cline { 2 - 2 } Faixas & $\mathbf{N}$ & $\%$ Pacientes & \\
Etárias (Ano) & 4 & 12,1 & 12,1 \\
$0-2$ & 10 & 30,3 & 42,4 \\
$3-4$ & 5 & 15,2 & 57,6 \\
$5-6$ & 8 & 24,2 & 81,8 \\
$7-8$ & 5 & 15,2 & 97 \\
$9-10$ & 1 & 03,0 & 100 \\
$11-12$ & 33 & 100 & 100 \\
Total & 1 & \\
\hline
\end{tabular}

Tabela 2 - Comparação entre RAST positivo e RAST negativo para látex em 30 pacientes

\begin{tabular}{|c|c|c|c|c|c|}
\hline & $\begin{array}{c}\text { Látex } \\
\text { positivo }\end{array}$ & $\begin{array}{c}\text { Látex } \\
\text { negativo }\end{array}$ & $\begin{array}{l}\text { Odds } \\
\text { Ratio }\end{array}$ & $\begin{array}{c}\text { Intervalo de } \\
\text { Confiança }\end{array}$ & $\begin{array}{l}\text { Valor } \\
\text { do } P\end{array}$ \\
\hline $\begin{array}{l}\text { Número de pacientes } \\
\text { Sexo, FM } \\
\text { Idade (anos) } \\
\text { No de cirurgias } \\
\text { DVP } \\
\text { Atopia } \\
\text { Cateterizacãoo diária } \\
\text { Enemas dírios } \\
\text { Hx de eczema atópico } \\
\text { Hx de afastamento do látex } \\
\text { Hx de reações imediatas ao látex } \\
\text { Hx de alergia alimentar } \\
\text { Hx de alergia a banana } \\
\text { RAST para banana positivo }\end{array}$ & $\begin{array}{c}14 \\
8 / 6 \\
4.8 \pm 2 \\
4 \pm 1.1 \\
13 \\
8(57.1 \%) \\
7(50 \%) \\
- \\
1(7.1 \%) \\
2(14.3 \%) \\
4(28.6 \%) \\
1(7.1 \%) \\
1(7.1 \%) \\
2(14.3 \%)\end{array}$ & $\begin{array}{c}16 \\
11 / 5 \\
5.6 \pm 3.3 \\
3.3 \pm 1.2 \\
15 \\
4(25 \%) \\
9(56.2 \%) \\
\mid(6.7 \%) \\
\mid(6.3 \%) \\
\mid(6.7 \%) \\
2(12.5 \%) \\
\mid(6.7 \%) \\
\mid(6.7 \%) \\
2(13.3 \%)\end{array}$ & $\begin{array}{c}0.87 \\
4.0 \\
0.78 \\
0 \\
1.15 \\
2.5 \\
2.8 \\
1.15 \\
0.87 \\
1.08\end{array}$ & $\begin{array}{c}0.01-73.2 \\
0.68-25.34 \\
0.15-4.10 \\
0-6.05 \\
0.01-96.53 \\
0.11-157.15 \\
0.31-35.47 \\
0.01-96.53 \\
0.01-73.2 \\
0.07-17.13\end{array}$ & $\begin{array}{l}\text { n.s. } \\
\text { n.s. } \\
\text { n.s. } \\
\text { n.s. } \\
\text { n.s. } \\
\text { n.s. } \\
\text { n.s. } \\
\text { n.s. } \\
\text { n.s. } \\
\text { n.s. } \\
\text { n.s. } \\
\text { n.s. } \\
\text { n.s. }\end{array}$ \\
\hline \multicolumn{6}{|c|}{$\begin{aligned} \text { Legenda }: \text { n.s. }=\text { não siggificativo } & \\
\text { DVP } & =\text { Derivaçäoventrículo-peritoneal } \\
H x & =\text { História }\end{aligned}$} \\
\hline
\end{tabular}

Os questionários continham perguntas sobre a presença de sinais e sintomas de alergia ao látex e reações cruzadas com alimentos. As crianças coletaram sangue para efetuarem lg $\mathrm{E}$ específica para látex e banana (exclusivamente pelo método RAST Pharmacia). Foi considerado positivo a lgE específica igual ou superior à classe I.

Trinta e três crianças, de baixo padrão socioeconômico, participaram do estudo. Doze crianças eram do sexo masculino e 21 do feminino, com idades desde 21 dias a 12 anos. Os responsáveis pelos pacientes foram inquiridos sobre a freqüência de manipulações retais e cateterizações urinárias realizadas nos menores, a presença de derivação ventrículo-peritoneal, o número de cirurgias a que foram submetidos, a história de problemas durante os atos cirúrgicos, a presença de atopia, história familiar de alergia, presença de sinais e sintomas de alergia alimentar, alergia a drogas e ao látex.

Foi coletado sangue de 30 crianças para efetuar lgE específica para látex (RAST Pharmacia), e de 29 para banana. O estudo foi aceito pelo comitê de ética da instituição. A análise estatística foi efetuada pelo Epiinfo 6. para calcular as médias, percentuais, e desvio padrão; o valor de $p$ considerado significativo foi $\leq 0.05$.

\section{Resultados}

Na distribuição por gênero, havia 21 meninas (63,6\%), e 12 meninos (36,4\%). Em relação à faixa etária, as idades dos pacientes variaram de zero a 12 anos, com média de cinco anos e desvio padrão (DP) de 2,6 anos (Tabela I).
Os dados clínicos e laboratoriais estão descritos na Tabela 2. Duas das 33 crianças (6,1\%) efetuavam a desimpactação manual fecal, e 18/33 (54,5\%) a cateterização urinária. O tempo de cateterização foi de 1-8 anos. Todas as crianças foram submetidas a pelo menos duas cirurgias, mas 10 crianças foram operadas mais de cinco vezes. A presença de derivações ventrículo-peritoneais ocorreu em 93,9\% das crianças. A história pessoal de alergia estava presente em 39,4\%, e a história familiar em 69.7\%. A dermatite atópica estava presente em 6,1\%. Urticária após ingesta de drogas ocorreu em quatro crianças (12,l\%). Apenas uma criança (3\%) apresentou problemas durante as cirurgias, com queixa de obstrução nasal. Quatro crianças (I2,1\%) apresentaram uma história positiva de alergia ao látex, três (9\%) com urticária de contato generalizada e angioedema, e estas três evitavam contato com produtos de látex.

Duas crianças $(6,1 \%)$ desenvolveram sinais de alergia alimentar. Uma criança apresentava urticária com inhame. A outra criança apresentava um relato de urticária com inhame, batata, banana, e leite de vaca. Também apresentava diarréia com carne de vaca, banana, batata, inhame e laranja.

A IgE foi positiva para látex em 12/30 $(46,6 \%)$ das crianças. De 29 crianças que efetuaram Ig E específica para banana, quatro (13,7\%) apresentaram resultado positivo, e 2/4 destas crianças também demonstraram positividade à lgE específica para látex (Tabela 3). As variáveis não foram estatisticamente significativas com a sensibilização ao látex e à banana.

\section{Discussão}

A idade da população estudada encontrava-se mais freqüentemente na faixa de 2-4 anos de idade (30,3\%), porque, para as crianças acima de 10 anos, o acesso ao hospital era muito difíicil. As dificuldades de transporte das crianças mais velhas, incapazes de andar, sem disponibilidade de cadeiras de rodas, podem justificar esta observação. Este viés de seleção, sem dúvida, interfere no resultado da pesquisa, porque quanto maior a faixa etária, maior a probabilidade de exposição e, conseqüentemente, de sensibilização.

O número de pacientes que eram submetidos à desimpactação manual fecal era muito baixo. Possivelmente esta observação provém 


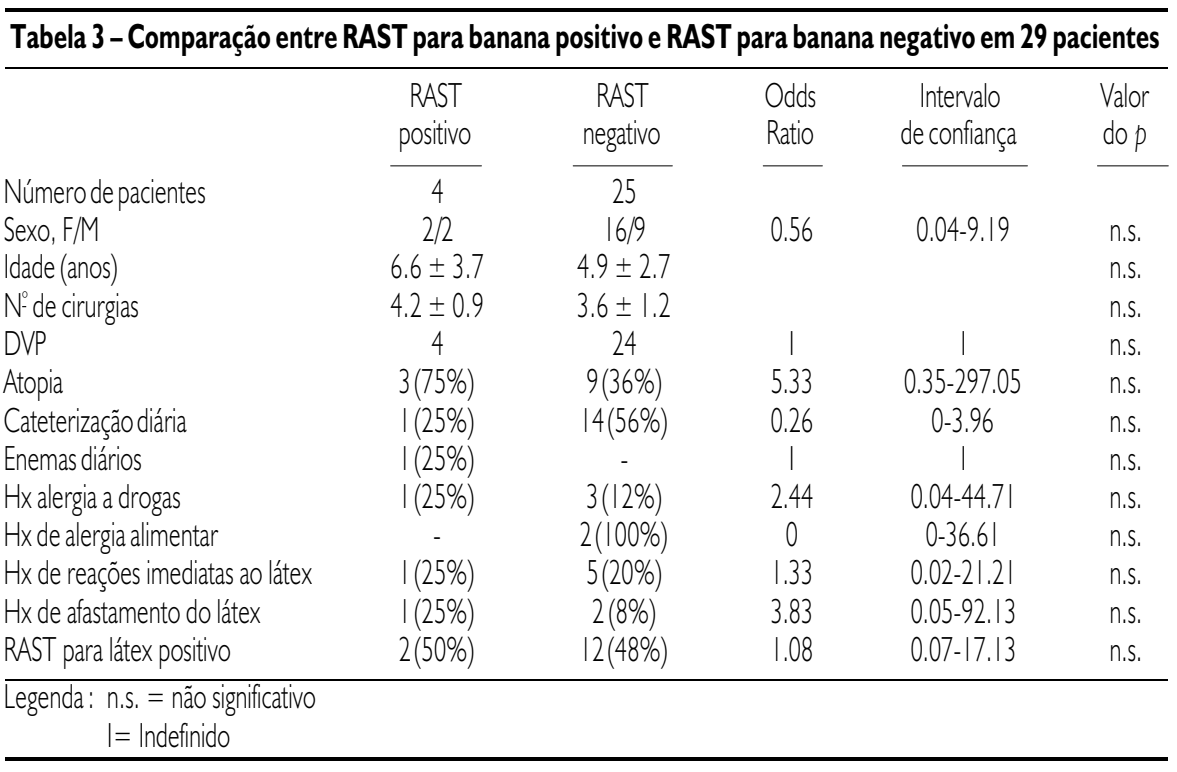

de limitações do nosso sistema nacional de saúde para acompanhar esta doença num país subdesenvolvido, o que pode justificar a mesma observação encontrada na Argentina ${ }^{4}$. 0 mesmo ocorreu com o tempo de cateterização urinária - a freqüência mais elevada foi de dois anos - e este achado interfere diretamente na freqüência de exposição e, subseqüentemente, de sensibilização ao látex.

A prevalência de alergia ao látex neste estudo foi de $12,1 \%$, resultado encontrado na literatura. $O$ diagnóstico de alergia ao látex repousa sobretudo na história clínica sugestiva $^{14}$ e é confirmado através de testes cutâneos e a presença de lgE específica (método do RAST) ${ }^{15}$ ou por um teste de provocação em caso de dúvida. A presença de relatos clínicos de alergia ao látex na literatura variou de zero a $72 \%$ '. Os diversos estudos ${ }^{1,2}$ avaliando pacientes com MMC através de história, testes cutâneos e/ou RAST vêm demonstrando variadas freqüências, de 2,97\% a 72\%. Os resultados obtidos nesta pesquisa se aproximam mais da freqüência observada nos EUA, através da história clínica, do que de outros países da América Latina, tais como a Venezuela (3,2\%) e a Argentina (0,99\%).

Uma das limitações do presente trabalho que poderia influir nos resultados negativos encontrados nas tabelas é o pequeno número de casos. É possível que uma amostra com maior número de casos evidenciasse certas correlações sugeridas por alguns valores elevados de OR nas Tabelas 2 e 3.
Só evitavam o látex as crianças que apresentavam uma história de urticária de contato que evoluía com edema palpebral após o uso de bolas de aniversário e luvas cirúrgicas. Esta observação nos leva a refletir que esta população está apenas tomando medidas preventivas de exposição ao látex quando o quadro é importante. Observamos que estas famílias não somente desconhecem a alergia ao látex, como também ignoram que se encontram no principal grupo de risco desta doença.

Não obtivemos informações sobre a reutilização de luvas e cateteres de uma parcela considerável das crianças. Este é um dado importante, principalmente para a comparação com os países desenvolvidos, e poderá ser uma das explicações para a diferença nas prevalências da alergia ao látex entre a América Latina, a Europa e EUA, visto que a lavagem freqüente dos produtos de látex facilita a perda do conteúdo protéico.

A sensibilização laboratorial ao látex ou à banana pode ser mais correlacionada, como encontrado em outros estudos, a um íntimo contato com o látex e à atopia. Esta observação é importante em países subdesenvolvidos, onde a banana é um alimento popular, visto que a sensibilização cruzada entre a banana e o látex é bem conhecida.

Não se encontrou causa aparente para a maior freqüência de alergia ao látex e à banana, neste estudo, em relação à observada na Venezuela ${ }^{3}$ e outros países em desenvolvimento. Além disso, observamos a presença de história de alergia ao inhame, um alimento usado principalmente entre as populações mais desfavorecidas no Brasil. Encontramos apenas uma referência a esta alergia na literatura (MEDLINE) ${ }^{16}$. Esta história pode demonstrar reações cruzadas com o látex ou outros alimentos. Para se comprovar esta possibilidade, outros estudos são necessários.

\section{Conclusóes}

A prevalência de histórias de reações caracterizadas como urticária de contato ao látex em crianças com mielomeningocele foi de $12,1 \%$, não tendo sido observados outros tipos de reações. As crianças que apresentavam história de urticária de contato com o látex que se generalizava e evoluía com edema palpebral relatavam um contato com o látex muito freqüente e íntimo.

Em 6, $1 \%$ das crianças houve um relato de alergia alimentar, sendo a urticária pelo inhame a reação alérgica mais freqüente. $A$ lgE foi positiva para látex em $46,6 \%$ das crianças. Das crianças que efetuaram lgE específica para banana, 13,7\% apresentaram resultado positivo. Não encontramos explicação para a nossa maior incidência de alergia ao látex e à banana em relação à observada em outros países da América Latina.

\section{Agradecimentos}

A Nazer S. C., do Laboratório Sérgio Franco, que efetuou a dosagem de lg Eespecífica para látex. A Oliveira L. F., do Laboratório Sérgio Franco, que efetuou a dosagem de lgE específica para banana.

\section{SUMMARY \\ LATEX AND BANANA aLlergies IN CHILDREN WITH MYELOMENINGOCELE IN RIO DE JANEIRO CITY}

PURPOSE. Recently, latex type I hypersensibility reactions were frequently described, mainly in children with myelomeningocele (MMC), which maintain earlier intimate and frequent contact with latex products. Allergic food cross-reactions are well known for many years. Nowadays, latex allergy is frequently associated with food allergies to avocado, banana, kiwi, grapefruit, papaya, chestnut and peach.

BACKGROUND. The objective of this study consisted in identifying the number of patients with myelomeningocele (MMC) and bananalatex clinico-laboratorial sensitization. 
Methods. Questionnaires were applied to 33 children with MMC, and blood was collected from 30 to perform latex lg E (RAST Pharmacia), and from 29 to banana $\lg E$. Specific $\lg E$ equal or above class I was considered positive.

RESULTS. Four children related histories of latex contact urticaria, and one child related a history of urticaria and diarrhea with banana. 1 4/30 (46.6\%) were latex Ig E positive, and 4/29 (13.7\%) to banana. There was no statistically significant association between variables.

Conclusions. We identified high prevalence of latex and banana allergies in patients with $M M C$, and we need more studies to analyze the prevalence of food-latex allergic cross-reactions in children with MMC. [Rev Assoc Med Bras 2004; 50(I): 83-6]

KEY wORDs: Banana allergy. Child. Latex allergy. Spina bifida.

\section{REFERÊNCIAS}

I. Turjanmaa K, Mäkinen-Kiljunen S, Reunala T, Alenius $\mathrm{H}$, Palosuo T. Natural rubber latex allergy - the european experience. Immunol. Allergy Clin North Am 1995; I (1):7 I-88.

2. Food and Drug Administration Medical Bulletin. Gelb, L. N., eds. 199|:July.
3. Capriles-Hulett A, Sánchez-Borges M, VonScanzoni C, Medina J. Very low frequency of latex and fruit allergy in patients with spina bifida from Venezuela: influence of socioeconomic factors. Ann Allergy Asthma Immunol 1995; 75(7):62-4.

4. Benhabib GO, Máspero JF, Kohan MJ, Gardes A, Poulin P. Latex allergy is no common in meningomyelocele in Argentina. J Allergy Clin Immunol 1995; 95(I part 2): I52.

5. Di Plácido P, Corbetta M, Docena G, Fernández R, Benítez P. Prevalência de hipersensibilidad al látec en pacientes com mielomeningocele. Rev Cir Infant 1997; 7(1):36-40.

6. Avila C, Navarro BEDR, Rosas MAV, Mongew JJLS. Reacción inmediata al látex: comparación entre la prueba cutánea y la determinación de Ig E por ELISA. Alergia Méx 200।; 48(4): I I0-4.

7. Fernández MR, Sandoval GF, Solano MO. Alergia al látex en una población de riesgo. Alergia Méx 1999; 46(4):93-6.

8. Brehler R, Theissen U, Mohr C, Luger T. "Latex-fruit syndrome": frequency of crossreacting Ig $\mathrm{E}$ antibodies. Allergy 1997; 52(4):404- 10.

9. Tucke J, Posch A, Baur X, Rieger C, RaulfHeimsoth M. Latex type I sensitization and allergy in children with atopic dermatitis. Evaluation of cross-reactivity to some foods. Pediatr Allergy Immunol 1999; 10(3): 160-7.
10. Mikkola K, Alenius H, Kalkkinen N. Molecular identification of cross-reacting allergens in natural rubber latex and banana [abstract]. J Allergy Clin Immunol 1997; 99(3):342.

I I. Kim KT, Hussain H. Prevalence of food allergy in 137 latex allergic patients. Allergy Asthma Proc 1999; 20(2):95-7.

12. Beezhold DH, Sussman GL, Liss GM, Chang NS. Latex allergy can induce clinical reactions to specific foods. Clin Exp. Allergy 1996; 26(4):416-22.

13. Delbourg MF, Guilloux L, Moneret Vautrin DA, Ville G. Hypersensitivity to banana in latex allergic patients. Identification of two major banana allergens of 33 and $37 \mathrm{kd}$. Ann Allergy Asthma Immunol 1996; 76(4):32 I-6.

14. Slater JE, Mostello LA, Shaer C. Type I hypersensitivity to rubber. Ann Allergy 1990; 65(5):4II-4.

15. Kelly KJ, Kurup VP, Zacharisen M. Skin and serologic testing in the diagnosis of latex allergy. J Allergy Clin Immunol 1993; 9।(6): $1140-5$.

16. Fine A.J. Hypersensitivity reaction to jicama (Pachyrhizus, yam bean). Ann Allergy 1991; 66(2): 173-4.

Artigo recebido: 06/02/03 Aceito para publicação: 31/07/03 